Innovation und Wissenschaft 
WISSENSCHAFT

UND

GESELLSCHAFT
Herausgegeben

vom Institut

für Theorie, Geschichte und Organisation der Wissenschaft der Akademie der Wissenschaften der DDR durch Günter Kröber

Band 23 


\section{Innovation und}

\section{Wissenschaft}

\section{Ein Beitrag zur Theorie und Praxis}

der intensiv erweiterten Reproduktion

Herausgegeben von

Günter Kröber und Harry Maier

AKADEMIE-VERLAG $\cdot$ BERLIN 1985 
Erschienen im Akademie-Verlag Berlin, DDR-1086 Berlin, Leipziger Straße 3-4 (C) Akademie-Verlag Berlin 1985

Lizenznummer : $202 \cdot 100 / 35 / 85$

Printed in the German Democratic Republic

Gesamtherstellung: VEB Druckerei „Thomas Müntzer“, 5820 Bad Langensalza Einbandgestaltung: Rolf Kunze

LSV 0305

Bestellnummer : $7540725(2162 / 23)$

02200 